\title{
SOME EXAMPLES OF RIGHT SELF-INJECTIVE RINGS WHICH ARE NOT LEFT SELF-INJECTIVE
}

\section{F. L. SANDOMIERSKI ${ }^{1}$}

ABSTRACT. Endomorphism rings of nonfinitely generated free right $R$-modules over a quasi-Frobenius ring $R$ are right self-injective but not left self-injective.

A well-known example of a right self-injective ring which is not left self-injective is the full ring of linear transformations of an infinite dimensional right vector space over a division ring, with linear transformations acting on the left of vectors, see e.g. [4].

The purpose of this note is to show that the class of rings which are right self-injective but not left self-injective includes the endomorphism rings of nonfinitely generated free right modules over a quasi-Frobenius ring.

All rings have unity element and all modules are unital.

THEOREM 1. Let $M_{R}$ be a right $R$-module, $R$ a ring, which is an infinite direct sum of nonzero submodule $\left\{M_{i} \mid i \in I\right\}, I$ some index set. For $S=\operatorname{Hom}\left(M_{R}, M_{R}\right)$ such that ${ }_{S} M_{R},{ }_{S} M$ is not an injective $S$-module.

Proof. Let $e_{i}: M_{R} \rightarrow M_{i}$ be the $i$ th projection of the module $M_{R}$ onto the submodule $M_{i}$, then $\left\{e_{i} \mid i \in I\right\}$ is an infinite set of orthogonal idempotents of $S$. Let ${ }_{S} A$ be the left ideal of $S$ generated by $\left\{e_{i} \mid i \in I\right\}$. Since $M_{i} \neq 0$ for each $i \in I$, choose $0 \neq x_{i} \in M_{i}$. Clearly there is an $S$ homomorphism $f:_{s} A \rightarrow_{S} M$ such that $e_{i} f=x_{i}=e_{i} x_{i}$. If $f$ were extendable to a homomorphism from ${ }_{s} S$ to ${ }_{s} M$, then it would be given by a right multiplication by some element of $M$. However for any element $x \in M, e_{i} x=0$ for all but finitely many $i \in I$, so $f$ is not extendable to ${ }_{s} S$, so ${ }_{s} M$ is not injective.

THEOREM 2. Let $R$ be a quasi-Frobenius ring and $F_{R}$ a nonfinitely generated free right $R$-module and $S=\operatorname{Hom}\left(F_{R}, F_{R}\right)$, so that ${ }_{S} F_{R}$, then $S$ is right self-injective but not left self-injective.

Proof. By [3, Theorem A], $F_{R}$ is injective since it is projective, hence for $A_{S},{ }_{S} F_{R}, F_{R}$

Received by the editors March 5, 1970.

AMS 1969 subject classifications. Primary 1650; Secondary 1690.

Key words and phrases. Self-injective ring, quasi-Frobenius ring.

1 This paper was written with partial support from NSF Grant GP-9564. 


$$
\begin{aligned}
\operatorname{Ext}_{S}^{1}\left(A_{S}, S_{S}\right) & =\operatorname{Ext}_{S}^{1}\left(A_{S}, \operatorname{Hom}\left(F_{R}, F_{R}\right)_{S}\right) \\
& \cong \operatorname{Hom}\left(\operatorname{Tor}_{1}\left(A_{S},{ }_{S} F\right)_{R}, F_{R}\right)
\end{aligned}
$$

by [2, Proposition VI. 5.1]. Since $F_{R}$ is a generator ${ }_{S} F$ is finitely generated projective by $\left[1\right.$, Theorem 2], hence $\operatorname{Tor}_{1}^{S}\left(A_{S},{ }_{S} F\right)=0$, so by (1), $\operatorname{Ext}_{S}^{1}\left(A_{S}, S_{S}\right)=0$ for each $A_{S}$, from which it follows that $S$ is right self-injective by [2, Proposition VI. 2.1a].

By Theorem $1,{ }_{s} F$ is not injective hence $S$ is not left self-injective since ${ }_{S} F$ is finitely generated projective (i.e. a direct summand of a finite number of copies of ${ }_{s} S$ ).

\section{REFERENCES}

1. G. Azumaya, Completely faithful modules and self-injective rings, Nagoya Math. J. 27 (1966), 697-708. MR 35 \#4253.

2. H. Cartan and S. Eilenberg, Homological algebra, Princeton Univ. Press, Princeton, N. J., 1956. MR 17, 1040.

3. C. Faith, Rings with ascending condition on annihilators, Nagoya Math. J. 27 (1966), 179-191. MR 33 \#1328.

4. B. Osofsky, Cyclic injective modules of full linear rings, Proc. Amer. Math. Soc. 17 (1966), 247-253. MR $32 \# 7604$.

University of Wisconsin, Madison, Wisconsin 53706 